\section{Resumen}

La exclusión es un fenómeno de gran magnitud que no sólo afecta a quienes están fuera del sistema educativo, sino también a quienes estando escolarizados son segregados, discriminados, o, no aprenden porque reciben una educación de baja calidad. La inclusión tiene diferentes interpretaciones, siendo muy frecuente su asimilación con la integración de las personas con discapacidad u otras con necesidades educativas especiales en la escuela común, cuando se trata de dos enfoques con una visión distinta. La educación inclusiva aspira a ofrecer una educación de calidad a toda la población, asegurando la plena participación y los logros de aprendizaje de todos los estudiantes, con especial énfasis en aquellos que, por diferentes razones, están excluidos o en riesgo de ser marginados.

La inclusión implica una transformación profunda de los sistemas educativos y de la cultura de las escuelas para que sean capaces de atender la diversidad de necesidades de aprendizaje del alumnado. Esto exige el desarrollo de políticas intersectoriales, con un enfoque de derechos, que aborden de forma integral los factores que generan exclusión dentro y fuera de los sistemas educativos; combinar estrategias globales para toda la población, enfatizando las de carácter preventivo, con medidas focalizadas para los colectivos en situación de mayor vulnerabilidad; avanzar desde estrategias homogéneas hacia políticas que consideren la diversidad con cohesión social, y el desarrollo de currículos, y sistemas de evaluación inclusivos.

\section{Palabras clave}

Educación inclusiva, derecho a la educación, educación de calidad para todos, políticas de inclusión, no discriminación, equidad

\section{Abstract}

Exclusion is a large-scale phenomena affecting not only those who are out of the school system, but also those who, in spite of acquiring formal schooling, are segregated, discriminated, or are unable to learn due to the poor quality of education they receive. Inclusion has multiple interpretations and is frequently associated with integrating the disabled or others with special education needs, in the ordinary school; however we are dealing with two approaches with different views. Inclusive education aims at offering quality education to all, ensuring full participation and learning achievements of all students, with special emphasis on those who, for various reasons, are excluded or are at risk of being marginalized.

Inclusion implies an in depth transformation of educational systems and the school culture in order to address the diversity of student learning needs. This requires designing intersectoral policies, on a rightsbased approach, for an integral treatment of factors that generate exclusion inside and outside educational systems; to combine global strategies for the population as a whole with emphasis on those of a preventive nature, and measures focused on most vulnerable groups; to progress from homogeneous strategies towards policies that envisage diversity with social cohesion, development of curricula, and inclusive educational systems.

\section{Key words}

Inclusive education, right to education, quality education for all, inclusion policies, no-discrimination, equity 


\title{
Haciendo efectivo el derecho a una educación de calidad sin exclusiones
}

\author{
Rosa Blanco Guijarro²
}

El presente artículo está estructurado en cuatro partes. En el primer apartado se presenta un breve panorama de la exclusión educativa y social en los países de América Latina y el Caribe. En el segundo, se aborda el significado del movimiento de la educación inclusiva desarrollando sus principales características y señas de identidad. El tercer apartado describe la importancia de la inclusión para hacer efectivo el derecho a una educación de calidad para todos y los sentidos de este movimiento: la inclusión como un asunto de derechos humanos, la inclusión como un medio para brindar una educación de calidad para todos; la inclusión como un elemento de justicia social y como un medio para aprender a ser y a vivir juntos. En el último apartado se señalan algunos lineamientos de políticas para avanzar hacia el desarrollo de escuelas y sistemas educativos más inclusivos.

\section{Exclusión educativa y social en América Latina y el Caribe}

La exclusión educativa y social son fenómenos crecientes tanto en los países desarrollados como en desarrollo, y son especialmente críticos en América Latina, que se caracteriza por tener sociedades altamente fragmentadas, debido a la persistencia de la pobreza y a la desigual distribución de recursos materiales y simbólicos. La exclusión social es un fenómeno que va más allá de la pobreza, ya que tiene que ver con

\footnotetext{
${ }^{1}$ Texto recibido el 29 de febrero de 2008, evaluado el 9 de mayo y 3 de junio, y arbitrado el 9 de junio de 2008.

2 Especialista regional en educación inclusiva y educación de la primera infancia. Directora (en funciones) OREALC/UNESCO, Coordinadora de la red UNESCO de Innovaciones Educativas: INNOVEMOS. rblanco@unesco.cl; r.blanco@unesco.cl
} 
la dificultad de desarrollarse como persona, la falta de oportunidades para elegir y desarrollar el proyecto de vida, la ausencia de participación en la sociedad y de acceso a sistemas de protección y de bienestar. Todos estos factores conducen finalmente a un sentido de no pertenencia y a la ruptura del vínculo con la sociedad.

Una de las principales funciones de la educación es contribuir a la reducción de las desigualdades y a la cohesión social, pero cabe preguntarse si las políticas educativas que se están desarrollando están logrando este objetivo. La mayoría de los países adoptan los principios de la Declaración de Educación para Todos, pero en la práctica existen distintos factores que excluyen y discriminan a numerosos alumnos del sistema educativo. Los niños y niñas llegan a la escuela en condiciones muy diferentes según el nivel de ingresos y el capital cultural de sus familias, sus posibilidades de acceso a las tecnologías de la información y comunicación, o las características y recursos de las comunidades en las que viven, entre otros. Estas desigualdades de origen son reproducidas e incluso acentuadas en el ámbito escolar debido a factores como; escuelas muy alejadas y/o incompletas, que limitan el acceso a la educación y la continuidad de estudios; la segmentación de las escuelas, las que tienen mayores necesidades cuentan con docentes menos cualificados y menores recursos; y procesos educativos que tienden a discriminar a aquellos estudiantes con un capital cultural distinto al dominante en la escuela.

Los avances logrados acompañados de medios efectivos que garanticen la permanencia en el sistema escolar y la satisfacción efectiva de las necesidades básicas de aprendizaje de todos los estudiantes. Los sistemas educativos atrasan o expulsan a lo largo de la educación primaria a 4 de cada 10 niños que ingresaron oportunamente en el primer grado (OREALC/ UNESCO, 2007b).

En aquellos países que cuentan con datos desagregados, los niños y jóvenes que viven en situación de pobreza, en la zona rural, de pueblos originarios o afrodescendientes, de familias migrantes o con discapacidad son lo que presentan tasas más altas de repetición y abandono escolar, y los que menos acceden a la educación, secundaria y terciaria. El promedio regional de personas entre 15 y 24 años de la zona rural que tiene al menos diez años de estudios es tan solo de un $20 \%$ frente a un $50 \%$ de la zona urbana, y las tasas de conclusión de estudios de los estudiantes que provienen de los pueblos originarios muestran disparidades más pronunciadas que las observadas entre zona rural y urbana, y se hacen muy marcadas en el paso de la educación primaria a la secundaria ${ }^{3}$ (OREALC/UNESCO, 2007 b).

En relación con el género, salvo algunos países, no existen diferencias significativas en el acceso a la educación pero sí en la conclusión de estudios. Mientras que en los países de América Latina son mayores las tasas de abandono y reprobación de las

\footnotetext{
${ }^{3}$ El valor más alto para la alta secundaria (Chile) sólo llega al 60\%; mientras el más bajo (Nicaragua) es equivalente a 7,1\%. En cinco países (Ecuador, Paraguay, Guatemala, Nicaragua y Panamá), menos de 1 de cada tres personas originarias de 20 a 24 años ha logrado concluir la alta secundaria (OREALC/ UNESCO, 2007b).
} 
niñas de zona rural y comunidades indígenas, en los del Caribe y algunos latinoamericanos son los varones quienes abandonan antes la escuela y tienen un menor nivel de desempeño académico. En los escasos países donde existen estadísticas confiables queda de manifiesto que los más excluidos son los niños con discapacidad porque existe un alto porcentaje que no recibe ningún tipo de educación, especialmente los que tienen discapacidades más severas. Aunque la tendencia es promover la integración de estos alumnos en la escuela común, la gran mayoría está escolarizada en centros de educación especial, por lo que también son los más discriminados.

Los estudios internacionales sobre logros de aprendizaje muestran que tan sólo una minoría de alumnos, en su mayoría provenientes de escuelas privadas, logra desarrollar las competencias básicas para insertarse en la sociedad (OREALC/ UNESCO, 2001). La fragmentación de los sistemas educativos da lugar a circuitos muy diferenciados en los que existen escuelas muy homogéneas y de muy distinta calidad. Las escuelas públicas, especialmente las que están situadas en contextos muy desfavorecidos, cuentan con menores recursos y con personal menos cualificado, a pesar de tener mayores necesidades.

El debilitamiento de la escuela pública en muchos países es preocupante, dado su rol histórico en asegurar la igualdad y promover la integración social. La creciente participación del sector privado en la educación ${ }^{4}$ y la presencia de modelos que consideran ésta como un servicio o producto regulado por las reglas del mercado, está teniendo como consecuencia que los límites entre lo público y lo privado sean cada vez menos nítidos y que el Estado no se responsabilice en algunos países de garantizar una educación gratuita y de calidad a todos sus ciudadanos. (OREALC/UNESCO, 2007a)

\section{El significado del movimiento de la educación inclusiva}

El movimiento de la inclusión ha surgido con fuerza en los últimos años para enfrentar los altos índices de exclusión y discriminación presentes en la mayoría de los sistemas educativos del mundo. Tal como se ha visto en el anterior apartado, la educación no está siendo capaz de contribuir a reducir la brecha social ni las desigualdades educativas. El término inclusión tiene diferentes significados en los países. Con frecuencia se piensa que está relacionada con la participación de los estudiantes con discapacidad u otros con necesidades educativas especiales en la escuela común; es decir, se está asimilando el movimiento de inclusión con el de integración, cuando se trata de dos enfoques con una visión y foco distintos. Esta confusión tiene como consecuencia que las políticas de inclusión se consideren como una responsabilidad de la educación especial, limitándose el análisis de la totalidad de exclusiones y discriminaciones que se dan al interior de los sistemas educativos.

\footnotetext{
${ }^{4}$ El gasto privado en educación es de un promedio del $1.3 \%$ del PIB en los países de la OECD, mientras que es de un $6 \%$ en Jamaica; $3.6 \%$ en Colombia; $2.6 \%$ en Chile y $2 \%$ en Perú (Tomasevski, 2006).
} 
La exclusión en educación afecta no sólo a aquellos que nunca han accedido a la escuela o son expulsados tempranamente de ella, sino también a quienes estando escolarizados sufren discriminaciones o son segregados por su origen social y cultural, por su género, o sus niveles de rendimiento, y a quienes no logran aprender porque acceden a escuelas de muy baja calidad. La exclusión educativa es por tanto un fenómeno de gran magnitud que amerita una revisión profunda de los factores que la generan, internos y externos a los sistemas educativos, y el desarrollo de políticas intersectoriales que los aborden de forma integral (Blanco, en prensa).

La UNESCO (2005) define la educación inclusiva como un proceso orientado a responder a la diversidad de los estudiantes incrementando su participación y reduciendo la exclusión en y desde la educación. Está relacionada con la presencia, la participación y los logros de todos los alumnos, con especial énfasis en aquellos que, por diferentes razones, están excluidos o en riesgo de ser marginados, constituyendo un impulso fundamental para avanzar en la agenda de Educación para Todos.

La presencia se refiere al acceso y permanencia de los estudiantes en las escuelas de su comunidad. La participación está relacionada con la calidad de las experiencias educativas, asegurando la máxima participación de los estudiantes en el currículo y las actividades educativas y en los procesos de toma de decisiones que afectan sus vidas y el funcionamiento de la escuela. Este aspecto es sumamente importante porque muchos estudiantes acceden a las escuelas comunes pero no tienen una participación efectiva en la vida escolar. Finalmente, los logros incluyen los resultados de aprendizaje alcanzados por los estudiantes en relación con el currículo. La inclusión aspira a que todos aprendan lo máximo posible, desarrollando de forma equilibrada las competencias necesarias para insertarse en la sociedad, desarrollar su proyecto de vida y ejercer la ciudadanía mundial y local.

La inclusión se caracteriza por los siguientes aspectos:

- Aspira a ofrecer una educación de calidad para todos pero se preocupa especialmente de aquellos que, por diferentes causas, están excluidos o en riesgo de ser marginados. Si bien los estudiantes con discapacidad son los más excluidos existen otros grupos que enfrentan múltiples barreras para acceder y permanecer en la escuela y tener éxito en su aprendizaje. Estos pueden variar de un país a otro pero generalmente los niños y niñas de pueblos originarios o afrodescendientes, que viven en contextos aislados o de pobreza, niños de familias migrantes o sin certificado de nacimiento, portadores de VIH/SIDA o afectados por conflictos armados o la violencia, se encuentran en una situación de desventaja educativa y de vulnerabilidad social. Es una responsabilidad moral hacer un seguimiento de los grupos en una posición de mayor riesgo para ofrecerles lo más tempranamente posible las ayudas y recursos que necesitan para superar su situación de desventaja. La falta de estrategias adecuadas para atender las distintas necesidades de los estudiantes se refleja en los altos índices de repetición y abandono y en los bajos niveles de aprendizaje. 
- Implica una visión diferente de la educación basada en la diversidad y no en la homogeneidad. Todos los seres humanos compartimos una serie de características comunes que nos asemejan pero tenemos, al mismo tiempo, cualidades distintas que nos hacen ser únicos e irrepetibles. La larga tradición de concebir las diferencias desde criterios normativos; lo que falta o se distancia de lo normal o de lo frecuente, ha conducido a la creación de opciones distintas para aquellos categorizados como diferentes. La valoración negativa de las diferencias y los estereotipos conducen a la exclusión y la discriminación.

Desde la perspectiva de la educación inclusiva las diferencias están dentro de lo normal porque son consustanciales a la naturaleza humana, y se conciben como algo positivo que enriquece los procesos de aprendizaje. Cada estudiante se enfrenta, de manera distinta, a las experiencias de aprendizaje como consecuencia de su origen social y cultural, su género, y sus características individuales en cuanto a capacidades, motivaciones, ritmo y estilo de aprendizaje, haciendo que no haya dos alumnos idénticos. En el Informe de la Comisión Delors (UNESCO, 1996) se invita a adoptar el respeto a la diversidad como principio fundamental para combatir todas las formas de exclusión en la educación y para devolverle a la educación su rol central como crisol que contribuye a la armonía social.

Si las diferencias son algo normal entonces deben formar parte de la educación para todos, y no ser objeto de modalidades o programas segregados o diferenciados. La atención a la diversidad es una responsabilidad del sistema educativo en su conjunto, el cual enfrenta el desafío de proporcionar unos aprendizajes básicos para todos, que aseguren la igualdad, y de dar respuesta al mismo tiempo a las necesidades específicas de cada uno, sin que esto conduzca a la discriminación o la desigualdad.

- El foco de atención de la inclusión es la transformación de los sistemas educativos y de las escuelas para que sean capaces de atender la diversidad. Lograr el equilibrio entre lo común y lo diverso implica cambios profundos en las escuelas, dada la larga tradición de atender preferentemente lo supuestamente común. A diferencia de la integración, donde el énfasis ha estado en la atención de las necesidades específicas de los alumnos integrados, manteniendo inalterables los sistemas educativos, la principal preocupación de la inclusión es transformar las culturas, las prácticas y la organización de las escuelas para optimizar el aprendizaje y la participación de todos. Desde esta perspectiva ya no son los alumnos quienes se tienen que adaptar a la oferta educativa disponible, sino que el currículo y la enseñanza se diversifican y se adaptan a sus necesidades educativas.

- Se preocupa de identificar y minimizar las barreras que enfrentan los estudiantes para acceder y permanecer en la escuela, participar y aprender. El progreso de 
los alumnos no depende sólo de sus características personales sino del tipo de oportunidades y apoyos que se le brindan o no se le brindan, por lo que el mismo estudiante puede tener dificultades de aprendizaje y de participación en una escuela y no tenerlas en otra. La escasez de recursos, la rigidez de la enseñanza, la falta de pertinencia de los currículos, la formación de los docentes, la falta de trabajo en equipo o las actitudes discriminatorias son algunos de los factores que generan exclusión en las escuelas. (Blanco, 2006)

Desde el enfoque de la inclusión el problema no es el niño sino el sistema educativo y las escuelas. Las barreras al aprendizaje y la participación aparecen en la interacción entre el alumno y los distintos contextos: las personas, políticas, instituciones, culturas y las circunstancias sociales y económicas que afectan sus vidas. En este sentido, las acciones han de estar dirigidas principalmente a eliminar las barreras físicas, personales e institucionales que limitan las oportunidades de aprendizaje y el pleno acceso y participación de todos en las actividades educativas (Booth \& Ainscow, 2000). La identificación de barreras requiere recabar, cotejar y evaluar información proveniente de múltiples fuentes que permita planificar los cambios necesarios en las políticas y prácticas.

- La inclusión es un proceso que nunca está acabado del todo porque implica un cambio profundo de los sistemas educativos y de la cultura escolar. Las instituciones educativas tienen que revisar constantemente sus valores, organización y prácticas educativas para identificar y minimizar las barreras para el aprendizaje y la participación, buscando las estrategias mas adecuadas para dar respuesta a la diversidad y aprendiendo de las diferencias; por ejemplo, muchas escuelas acogen a estudiantes con discapacidad pero al mismo tiempo expulsan a otros por bajo rendimiento o problemas de adaptación. Esto nos muestra que han logrado superar actitudes de discriminación hacia un determinado colectivo pero permanecen otras barreras para otros estudiantes que es preciso identificar y minimizar.

- Sistemas de apoyo para todos los estudiantes y las escuelas que las precisen. Los docentes necesitan ayuda para atender la diversidad del alumnado. En el movimiento de la integración este apoyo es brindado por los profesionales de la educación especial, pero desde la perspectiva de la inclusión éstos son insuficientes porque sólo están preparados para atender un tipo de diferencias: las asociadas a las necesidades educativas especiales. Si se quiere avanzar hacia las escuelas inclusivas hay que pensar en el tipo de apoyo que necesitarán los docentes, qué perfiles profesionales deberían tener, cómo han de organizarse y cuáles serían sus funciones. Los apoyos han de trabajar de forma colaborativa con los docentes para resolver los problemas conjuntamente. 


\section{Los sentidos de la educación inclusiva}

La educación inclusiva es un movimiento con una filosofía clara que se basa en fundamentos éticos, sociales y educativos, los cuales se desarrollan a continuación.

\section{La educación inclusiva es un asunto de derechos humanos}

La educación es un bien público y un derecho humano del que nadie puede quedar excluido porque gracias a ella nos desarrollamos como personas y como sociedades. La educación es una poderosa herramienta que nos permite ejercer otros derechos como el acceso a un empleo digno, o ejercer la libertad de expresión haciendo posible el pleno ejercicio de la ciudadanía. Hacer efectivo el derecho a la educación requiere a su vez garantizar otros derechos como el de la salud, la nutrición, o el derecho de los niños a no trabajar.

El derecho a la educación en su sentido más amplio va más allá de la mera escolarización. Su pleno ejercicio exige que ésta sea de calidad, promoviendo el máximo desarrollo de las múltiples potencialidades de cada persona, a través de aprendizajes socialmente relevantes y experiencias educativas pertinentes a las necesidades y características de los individuos y de los contextos en los que se desenvuelven. El derecho a la educación es el derecho a aprender desde el nacimiento y a lo largo de toda la vida (OREALC/UNESCO, 2007a). El aprendizaje a lo largo de la vida requiere una transformación profunda de los sistemas educativos para que sean capaces de brindar variadas oportunidades y modalidades de ingreso o reingreso con el fin de acceder o completar estudios en cualquier momento de la vida. Este aspecto es especialmente sensible en América Latina dados los altos índices de abandono escolar.

Para que el derecho a una educación de calidad sea garantizado con justicia tiene que ser reconocido y aplicado igualitariamente a todas las personas, sin ningún tipo de discriminación. La inclusión de cualquier grupo pasa por una igualdad de derechos y por el respeto de sus libertades para lograr que todas las personas sientan que pertenecen y forman parte de la escuela y la comunidad. El sistema de Naciones Unidas, ha promovido diferentes convenciones y declaraciones para proteger los derechos de ciertos colectivos minoritarios o con menor poder dentro de la sociedad, en las que se considera la educación como uno de sus componentes fundamentales ${ }^{5}$.

En el ámbito de la educación el instrumento internacional más importante es la Convención contra la Discriminación en Educación ${ }^{6}$ (UNESCO, 1960). En ella

\footnotetext{
${ }^{5}$ Convención sobre la eliminación de todas formas de discriminación contra la mujer (ONU, 1979); Convención sobre la eliminación de toda forma de discriminación racial (1969); Declaración sobre los derechos de las personas pertenecientes a minorías nacionales o étnicas, religiosas y lingüísticas (1992). Convención para la protección y promoción de los derechos y la dignidad de las personas con discapacidad (ONU, 2006).

${ }^{6}$ Convention against Discrimination in Education (UNESCO, 1960).
} 
se considera la discriminación como cualquier distinción, exclusión, limitación o preferencia basada en la raza, sexo, lengua, religión, motivos políticos u otros tipos de opinión, origen social y económico, país de origen, que tiene como propósito o efecto: limitar a determinadas personas o grupos su acceso a cualquier tipo y nivel educativo; proporcionar a determinadas personas una educación con estándares inferiores de calidad; establecer o mantener sistemas educativos o instituciones separadas para personas o grupos; o inflingir a determinadas personas o grupos un trato incompatible con la dignidad humana.

Las prácticas discriminatorias atentan contra la dignidad de los estudiantes, en tanto sujetos de derechos, y profundizan la segregación y estratificación presentes en las sociedades porque limitan el encuentro entre estudiantes de diferentes contextos y culturas y con diferentes capacidades, afectando así la integración y la cohesión social que son funciones esenciales de la educación. Además, el hecho que determinadas escuelas sean excluyentes conlleva que aquellas que aceptan a todos, especialmente las públicas de contextos más desfavorecidos, terminen concentrando un alto porcentaje de estudiantes con mayores necesidades educativas que no pueden ser atendidas adecuadamente con los recursos disponibles. Se da entonces la paradoja que escuelas que reciben a todos, haciendo efectivo el derecho a la educación, se encuentran en una situación de desventaja, especialmente en aquellos sistemas donde los incentivos están asociados a los resultados de aprendizaje de los alumnos y cuando no existen recursos adicionales ni sistemas de apoyo para las escuelas con mayores necesidades (Blanco, en prensa).

Hacer efectivas la no discriminación y la plena participación requiere avanzar hacia escuelas más inclusivas en las que se acoja a todos los niños independientemente de sus condiciones personales, culturales o sociales; niños discapacitados y bien dotados, niños de la calle, de minorías étnicas, lingüísticas o culturales, de zonas desfavorecidas o marginales, lo cual plantea un reto importante para los sistemas educativos (UNESCO, 1994).

Es muy distinto acceder a una escuela homogénea o a una que de cabida a la diversidad de necesidades de aprendizaje. Las escuelas homogéneas impiden a los estudiantes aprender de otros con experiencias distintas a las propias, y no contribuyen al fortalecimiento de la ciudadanía ni a la democracia. Las escuelas heterogéneas, por el contrario, aseguran la igualdad de oportunidades, son más innovadoras, logran mejores aprendizajes y un mayor desarrollo profesional de los docentes (Ainscow, 2001). Los resultados de PISA $^{7}$ en el 2003 (OECD, 2004), muestran que

\footnotetext{
${ }^{7}$ Programa Internacional de Evaluación de Estudiantes en el que se evalúa el nivel de logro de los estudiantes de los países de la OECD (sigla en inglés de la Organización para la Cooperación y el Desarrollo Económico) en lenguaje, matemáticas y ciencias y otros aspectos como la motivación de los estudiantes, sus creencias y sus estrategias de aprendizaje. También se examinan las variaciones del desempeño en función del género y el contexto socioeconómico y se exploran algunos factores asociados a los niveles de logro. El estudio del año 2000 estuvo focalizado en lenguaje y el del año 2003, en matemáticas.
} 
la existencia de diferentes formas de selección académica no tiene una relación significativa con el nivel global del desempeño de los países, explicando entre un $6 \%$ y un $10 \%$ de la varianza de los resultados y, sin embargo, atentan contra la equidad. Finlandia, país con altos niveles de desempeño, se caracteriza por tener escuelas muy diversas, mostrando mayores diferencias en los resultados de aprendizaje al interior de las escuelas, que entre escuelas.

\section{La educación inclusiva aspira a ofrecer una educación de calidad para todos}

Ofrecer una educación de calidad para todos es indispensable para lograr la inclusión educativa y social, pero es preciso preguntarse qué cualidades debiera reunir para lograr este objetivo. La calidad de la educación tiene diferentes interpretaciones porque implica hacer un juicio de valor que está mediatizado por factores ideológicos y políticos, los sentidos que se le asignan a la educación, las diferentes concepciones sobre el desarrollo humano y el aprendizaje, y por los valores predominantes en una determinada cultura (OREALC/UNESCO, 2007a).

En muchos países se valora la calidad en función de criterios de eficacia y eficiencia, que siendo importantes son insuficientes desde la perspectiva de la inclusión. La educación es un proyecto cultural que se sustenta en una serie de concepciones y de valores respecto al tipo de sociedad que se quiere construir y del ideal de persona que se quiere desarrollar. Si anhelamos sociedades más justas y democráticas habrá que valorar la calidad de la educación no sólo por los logros de aprendizaje de los estudiantes, sino también por la distribución equitativa de los mismos. ¿Podríamos decir que una educación es de calidad si tan solo una minoría de la población logra aprender lo necesario para actuar en las diferentes áreas de la vida humana? Si aspiramos a construir sociedades pacíficas y respetuosas de las diferencias, ¿es posible hablar de una educación de calidad cuando ésta sólo refleja las aspiraciones de las clases y culturas dominantes? (Blanco, en prensa).

La Oficina Regional de Educación de la UNESCO para América Latina y el Caribe (OREALC/ UNESCO) ha establecido cinco dimensiones para definir una educación de calidad, desde la perspectiva de un enfoque de derechos $(2007 \mathrm{a})^{8}$ :

- Equidad. Significa que cada persona reciba los recursos y ayudas que requiere para participar y aprender a niveles de excelencia, de forma que la educación no reproduzca las desigualdades de origen de los estudiantes ni condicione sus opciones de futuro. No hay educación de calidad sin equidad ni equidad sin calidad.

\footnotetext{
${ }^{8}$ Estas dimensiones fueron adoptadas por los ministros de educación de América Latina y el Caribe, en la Declaración de la II Reunión Intergubernamental del Proyecto Regional de Educación para América Latina y el Caribe, Buenos Aires, 29 y 30 de marzo de 2007.
} 
- Relevancia. Una educación es de calidad si promueve el desarrollo de las competencias necesarias para participar en las diferentes áreas de la vida humana, afrontar los desafíos de la sociedad actual y desarrollar el proyecto de vida en relación con los otros. Desde la perspectiva de la UNESCO (1996) la educación ha de promover de forma equilibrada los cuatro pilares del aprendizaje: aprender a conocer, aprender a hacer, aprender a ser y a vivir juntos. Una educación relevante también ha de promover la educación y vivencia de los derechos humanos, y la participación.

- Pertinencia. Alude a la necesidad de que ésta sea significativa para personas de distintos estratos sociales y culturas, y con diferentes capacidades e intereses, de forma que puedan apropiarse de los contenidos de la cultura, mundial y local, y construirse como sujetos en la sociedad, desarrollando su autonomía, autogobierno y su propia identidad. Para que haya pertinencia, es fundamental que el currículo y los métodos de enseñaza sean flexibles, de forma que puedan atender las necesidades y características de los estudiantes y de los diversos contextos sociales y culturales.

- Eficacia. Implica analizar en qué proporción se logran o no garantizar los principios de equidad, relevancia y pertinencia expresados en metas. Esto implica analizar por ejemplo: en qué medida todos los estudiantes logran los aprendizajes establecidos en el currículo escolar y la plena participación; en qué medida las personas acceden a la educación, independientemente de su origen social, cultural, género o condiciones individuales o, en qué medida el currículo es pertinente para todos.

- Eficiencia. Significa que se asignen los recursos necesarios y se utilicen adecuadamente para las metas relacionadas con la equidad, la relevancia y la pertinencia.

\section{La inclusión es una cuestión de justicia social}

La educación inclusiva es antes que nada una cuestión de justicia e igualdad, constituyendo un medio para avanzar hacia sociedades más inclusivas, más justas y democráticas. Proporcionar a todos el acceso a una educación de calidad, y no sólo a las clases y culturas dominantes, y fortalecer las interacciones entre estudiantes de diferentes contextos sociales y culturas y con diferentes capacidades en la escuela, es una poderosa herramienta para lograr la cohesión social.

Desde un enfoque de derechos es un imperativo ético reducir las brechas sociales tanto materiales y simbólicas, como a nivel de libertades y oportunidades, en un marco de atención a la diversidad. Los talentos están repartidos por igual entre todas las clases sociales y culturas, lo que no están distribuidos por igual son los 
recursos y las oportunidades para que todas las personas puedan desarrollar sus capacidades. Existe una relación dialéctica entre educación inclusiva y equidad social; si bien la educación puede contribuir a una mayor igualdad y movilidad social, no es menos cierto que el éxito en la educación requiere una mínima equidad social que garantice las condiciones que hacen posible el aprendizaje. (Tedesco, 2005)

La calidad de la educación desde la perspectiva de la inclusión implica un equilibrio entre excelencia y equidad. Es decir, no se puede hablar calidad cuando tan sólo una minoría de estudiantes aprende lo necesario para insertarse en la actual sociedad del conocimiento y desarrollar su proyecto de vida. La tensión entre excelencia y equidad es objeto de controversia en todos los países del mundo y es posible observar tres situaciones respecto del equilibrio entre ambas dimensiones (Marchesi y Martin, 1998). En algunos países se prima la excelencia por sobre la equidad, promoviendo la competitividad entre los centros educativos, a través de incentivos por los resultados alcanzados, y la información pública de los mismos para que los padres puedan elegir la escuela para sus hijos. Este modelo no ha mostrado lograr una mayor calidad ni equidad en el conjunto del sistema, porque tan sólo un pequeño porcentaje de escuelas, donde se educan las elites, son de excelencia.

En otros países se prima la equidad por sobre la excelencia promoviendo la mayor igualdad posible entre las escuelas, a través de una alta regulación y centralización por parte del Estado, y el desarrollo de políticas de discriminación positiva para aquellas con mayores necesidades. En este enfoque es también difícil que la mayoría del alumnado logre los máximos niveles de aprendizaje, porque no se da cabida al desarrollo de propuestas educativas diferenciadas que se puedan ajustar a las distintas necesidades de las personas. Finalmente, hay países que buscan un equilibrio entre equidad y excelencia promoviendo ofertas diferenciadas, que den respuesta a distintas necesidades, pero estableciendo mecanismos de regulación por parte del Estado, que eviten la desigualdad, y proporcionando más recursos a los centros educativos con mayores necesidades.

Una educación es de calidad cuando logra la democratización en el acceso y la apropiación del conocimiento. La verdadera igualdad de oportunidades pasa por la igualdad de capacidades para actuar en la sociedad y por aumentar las posibilidades de las personas para optar y decidir (Artigas, 2003; Sen, 1999), que es una de las dimensiones del desarrollo humano. Los países enfrentan el desafío de generar las condiciones indispensables para ampliar la posibilidad de elección que se brindan a todos, atendiendo las aspiraciones de aquellos que tienen menor poder dentro de la sociedad para alcanzar el desarrollo del que todos forman parte.

La equidad comprende los principios de igualdad y diferenciación, lo cual significa proporcionar a cada persona los recursos y ayudas que requiere, en función de sus características y necesidades individuales, para que esté en igualdad de condiciones de aprovechar las oportunidades educativas. No es suficiente ofrecer igualdad oportunidades educativas, hay que crear las condiciones para que éstas sean 
aprovechadas por cada persona especialmente en países con grandes desigualdades educativas y sociales (Blanco, 2006).

La democratización en el acceso al conocimiento requiere necesariamente asegurar la equidad en el acceso y en los procesos educativos. En relación con la primera, el Estado tiene la obligación de proveer instituciones y programas educativos a lo largo del país y que éstos sean accesibles para todos. La accesibilidad involucra tres dimensiones: la accesibilidad física, escuelas que sean seguras y que estén a una distancia razonable, o modalidades que utilicen las nuevas tecnologías; la accesibilidad al currículo, medios y recursos que permitan el aprendizaje y la participación en las actividades educativas, como por ejemplo el aprendizaje en la lengua materna, o equipamientos específicos para alumnos con necesidades educativas especiales y; la accesibilidad económica, asegurando la gratuidad de la educación obligatoria (ONU/ECOSOC/UNESCO, 2003).

La igualdad de oportunidades en los procesos educativos implica un trato diferenciado, que no sea discriminatorio o excluyente, en lo que se refiere a los recursos financieros, materiales, humanos, tecnológicos, y pedagógicos. Por ejemplo, un currículo flexible e intercultural, recursos y apoyos adicionales para todos aquellos que los necesiten para avanzar en su aprendizaje, la asignación de los docentes más competentes a aquellas escuelas y/o grupos de alumnos que tienen mayores necesidades, calendario escolar adecuado a las distintas necesidades, aprender en la lengua materna, materiales educativos pertinentes a las diferentes realidades sociales y culturales (OREALC/ UNESCO, 2007a).

\section{La educación inclusiva es un medio fundamental para "aprender a vivir juntos y aprender a ser"}

La inclusión en la sociedad o en la escuela no puede hacerse a costa de negar o desvalorizar la identidad personal y cultural, porque no se logrará una verdadera participación ni un aprendizaje efectivo. Los estudiantes que pertenecen a los grupos sociales y culturales con menor vinculación a la cultura escolar tienen normas, valores, creencias, comportamientos y visiones del mundo que no son consideradas en los procesos de enseñanza y aprendizaje, lo cual conduce a un sentido de no pertenencia y a un menor progreso que en muchos casos termina en el abandono de la escuela. La desvalorización de lo que aportan los estudiantes dificultará que realicen aprendizajes significativos, desmotivándose al no percibir la pertinencia de los contenidos en relación consigo mismos y con su contexto.

Una de las finalidades que se le atribuye a la educación en los diferentes instrumentos de carácter internacional es la de fomentar la comprensión, la tolerancia y las relaciones entre todas las naciones, grupos religiosos o raciales, y el mantenimiento de la paz. Para lograr este objetivo es imprescindible el desarrollo de escuelas inclusivas que eduquen en y para la diversidad, desarrollando valores y actitudes de solidaridad y valoración de las diferencias, reconociendo al otro como 
un otro válido y legítimo, y respetando los valores del pluralismo, la comprensión mutua y la paz; es decir, aprender a vivir juntos. La percepción y la vivencia de la diversidad permiten, por otra parte, construir y reafirmar la propia identidad y distinguirse de otros.

La formación de los estados nacionales en América Latina y el Caribe se constituyó sobre la base de la homogeneidad y la construcción de la identidad nacional, avasallando las identidades de las etnias originarias, afrodescendientes y otras minorías culturales. Desde la perspectiva de la inclusión, todas las culturas tienen igual valor (no hay jerarquía o superioridad entre culturas) y se considera que las diferencias culturales enriquecen los procesos de aprendizaje. Una escuela inclusiva tiene que lograr un equilibrio entre lo común y lo diverso, promoviendo aprendizajes que permitan el ejercicio de la ciudadanía mundial y local para que las personas puedan moverse con libertad en la sociedad actual. Un énfasis excesivo en lo local limita enormemente las posibilidades de insertarse en un mundo cada vez más globalizado, pero, al mismo tiempo, el desconocimiento de las experiencias de vida de los estudiantes puede generar dificultades de aprendizaje y de participación, al no sentirse acogidos ni valorados en la escuela.

La educación no sólo tiene la finalidad de socializar a los individuos a través de la apropiación de los contenidos de la cultura en la que están inmersos, sino que también ha de contribuir a la individuación de cada sujeto en la sociedad con su propia identidad. El ser humano se realiza plenamente como miembro de una comunidad y una cultura, pero también en el respeto a su individualidad, por lo que otro aspecto fundamental de la educación ha de ser aprender a ser que es fundamental para conocerse y valorarse a sí mismo y construir la propia identidad, para actuar con creciente capacidad de autonomía, de juicio y de responsabilidad personal en las distintas situaciones de la vida (UNESCO, 1996).

Aprender a ser demanda hacer efectivo el derecho a la propia identidad respetando a cada uno como es. Este derecho supone un conjunto de atributos, tanto de carácter físico como los referidos a la personalidad, que están mediatizados por el grupo social y cultural de referencia. El fortalecimiento de la propia identidad debe ir de la mano de la apertura y el diálogo con otras culturas diferentes para no caer en fundamentalismos que limiten el desarrollo de las personas y comunidades. El conocimiento de las demás culturas conduce a una doble toma de conciencia: la de la singularidad de la propia cultura pero también la de la existencia de un patrimonio común de toda la humanidad (UNESCO, 1996).

\section{¿Cómo avanzar hacia sistemas educativos y escuelas más inclusivas?}

El desarrollo de escuelas inclusivas requiere un cambio sistémico y profundo de los sistemas educativos que afecta a las concepciones y actitudes, a las políticas y a las prácticas de las escuelas. En este apartado se señalan algunos lineamientos 
de política educativa que pueden contribuir a lograr una mayor inclusión educativa y social ${ }^{9}$.

\section{Políticas con enfoque de derechos y participación social y fortalecimiento del rol del Estado como garante del derecho a la educación}

Considerar la educación como derecho, y no como un mero servicio o una mercancía, tiene implicaciones jurídicas para los gobiernos que tienen la obligación de respetar, asegurar, proteger y promover este derecho. El desarrollo de escuelas inclusivas sólo será posible si existe una apuesta política clara y marcos legales que establezcan derechos y responsabilidades y la provisión de los recursos necesarios. Una política de inclusión requiere asimismo una verdadera movilización política y colectiva, promoviendo la participación y el diálogo informado con los distintos actores involucrados, con el fin de identificar y analizar sus necesidades, establecer prioridades y considerar sus puntos de vista para que se apropien del sentido de las políticas y contribuyan a su puesta en práctica.

Las políticas de descentralización y de autonomía de las escuelas y la creciente privatización de la educación han debilitado el rol garante y regulador del Estado en el ejercicio del derecho a la educación de todos sus ciudadanos. La descentralización es necesaria porque dar el mismo trato a escenarios desiguales atenta contra la equidad; sin embargo, es preciso hacer un debate a fondo sobre los modelos de descentralización que se están implementando porque en muchos casos están acentuando las desigualdades y están debilitando el rol del Estado. Con frecuencia, la descentralización no va acompañada de los recursos necesarios ni del fortalecimiento de las capacidades para que los actores locales puedan desarrollar las nuevas funciones.

Un rol garante obliga a los estados a: proveer escuelas y programas suficientes a lo largo del país que sean accesibles a todos; establecer estándares de calidad para todas las escuelas y programas; fortalecer la oferta pública y mejorar su calidad; y establecer normativas que eviten la discriminación y fortalecer los sistemas de garantía existentes para exigir el derecho a la educación y a la no discriminación ${ }^{10}$.

Hacer efectivas las garantías constitucionales para una educación gratuita de calidad exige una firme voluntad política que se refleje en la asignación de los recursos necesarios. Al respecto, los ministros de educación de la región ${ }^{11}$ acordaron la

\footnotetext{
${ }^{9}$ Algunas políticas fueron adoptadas por los Ministros de Educación de América Latina y el Caribe en la Segunda Reunión Intergubernamental del Proyecto Regional de Educación, PRELAC II, Buenos Aires, 29 y 30 de marzo de 2007.

${ }^{10}$ Estas funciones fueron adoptadas en las recomendaciones de la II Reunión del Comité Intergubernamental del Proyecto Regional de Educación para América Latina y el Caribe (EPT/PRELAC), Buenos Aires, Argentina, 29 y 30 de marzo de 2007, excepto la de fortalecer la escuela pública y mejorar su calidad.

11 |l Reunión del Comité Intergubernamental del Proyecto Regional de Educación para América Latina y el Caribe (EPT/PRELAC), Buenos Aires, Argentina, 29 y 30 de marzo de 2007.
} 
necesidad de llevar a cabo pactos o acuerdos fiscales para lograr un incremento de los fondos públicos en educación y una revisión de la asignación y gestión de dichos recursos que sea coherente con un enfoque de derechos. La distribución equitativa debe ser la preocupación central en la asignación de los recursos, considerando los costos que supone ofrecer una educación de calidad según las distintas necesidades de las personas y de los contextos en los que se desarrollan y aprenden.

\section{Avanzar desde políticas sectoriales y fragmentadas hacia políticas integrales e intersectoriales}

Las políticas educativas por sí solas no crean las oportunidades para acceder al capital social y cultural necesario, siendo indispensable desarrollar políticas intersectoriales que aborden de forma integral los factores que generan exclusión, discriminación o desigualdad. A la educación le corresponde eliminar o minimizar las barreras del sistema escolar que limitan la igualdad en el acceso a los conocimientos. Pero, al mismo tiempo, es necesaria la implementación de políticas económicas y sociales que aborden las causas que generan desigualdad fuera de los sistemas educativos garantizando las condiciones mínimas para el aprendizaje. La articulación de políticas de inclusión con las políticas generales de desarrollo y de erradicación de la pobreza puede tener un impacto significativo en la inclusión.

Los países encuentran dificultades para la coordinación interinstitucional e intersectorial, por lo que es preciso buscar estrategias que permitan la articulación e integración real de distintas acciones, desde un marco conceptual común pero con funciones y servicios complementarios. Las políticas intersectoriales permiten coordinar las metas y estrategias para asegurar la inclusión en los diferentes niveles educativos, optimizar los recursos e integrar los elementos clave de las políticas de inclusión en las agendas de cada sector para asegurar que sean consideradas en los procesos de toma de decisiones (Blanco, en prensa).

\section{Mayor énfasis en medidas de carácter preventivo}

Una política de inclusión debería ser una estrategia de prevención y no tanto de solución correctiva a problemas que ya han hecho aparición. Existe una serie de ventanas de oportunidad para el aprendizaje que sólo se abren de par en par en los primeros años, por lo que es fundamental que todos los niños comiencen la vida en igualdad de condiciones (UNESCO, 2004).

Las políticas de equidad que se están desarrollando en América Latina no están teniendo los resultados deseados porque los niños inician la educación formal en condiciones muy desiguales, por ello es necesario invertir más en la educación y cuidado de la primera infancia y en la educación de los padres. Como muy bien expresa Van Der Gaag (2000) el desarrollo temprano de cada niño está relacionado con el desarrollo humano de un país, por lo que invertir en programas de educación y cuidado de la primera infancia es el comienzo natural de las políticas y programas 
de desarrollo humano. Elevar el nivel de formación de los padres es otro elemento fundamental para la prevención, dada su gran influencia en el aprendizaje de los niños, razón por la cual es preciso fortalecer las actividades orientadas a la alfabetización y educación de adultos.

Otra medida de carácter preventivo es el desarrollo de sistemas de apoyo que puedan colaborar con las escuelas y programas en la identificación y atención precoz de las dificultades de aprendizaje de los alumnos, que suelen ser consecuencia de una mala enseñanza. La mayoría de los países cuenta, en mayor o menor medida, con profesionales que pueden ser muy valiosos para las políticas de inclusión: psicólogos, docentes de educación especial, terapeutas de lenguaje, trabajadoras sociales u orientadores. La eficacia de los apoyos es mayor cuando se establece un sistema que combina centros de recursos sectoriales con apoyos fijos en aquellas escuelas cuya población escolar tiene mayores necesidades, involucrando también la participación de otros sectores como salud y bienestar social.

\section{Combinar estrategias globales para toda la población con estrategias focalizadas en las necesidades de los colectivos en situación de mayor vulnerabilidad}

El interés por la equidad como objetivo de las políticas educativas, a través de estrategias de focalización, discriminación positiva o acción afirmativa, adquirió mayor fuerza en la década de los noventa, pero no fue la principal prioridad, dedicándose mayores recursos a la eficiencia y eficacia de los sistemas educativos, lo cual explicaría la persistencia de las desigualdades educativas. La equidad y la inclusión debieran ser una preocupación central en las decisiones de las políticas educativas de carácter general, definiendo acciones para atacar los factores que generan desigualdad en los sistemas educativos, y no reducirse a medidas de carácter periférico orientadas a corregir los efectos colaterales de políticas que no se inscriben en una lógica de justicia (García-Huidobro, 2005).

No obstante, en países con grandes desigualdades educativas y sociales es preciso combinar políticas globales orientadas a toda la población con estrategias de focalización ya que éstas ofrecen resultados positivos en términos de proveer los recursos y la atención adecuados a las necesidades de cada uno, siempre y cuando haya una complementariedad con las políticas generales y no sean homogéneas. En general las políticas de focalización o discriminación positiva se caracterizan por ofrecer lo mismo a todos sus destinatarios, lo cual limita su impacto en obtener los resultados deseados.

\section{Avanzar desde estrategias homogéneas y estandarizadas hacia políticas que consideren la diversidad con cohesión social}

La educación tiene el imperativo ético de asegurar la igualdad sin que ello signifique uniformidad, para no reproducir las desigualdades y exclusiones presentes en la 
sociedad. Ofrecer una educación de calidad sin exclusiones depende en gran medida del grado de ajuste entre la oferta educativa y las distintas necesidades educativas del alumnado. El mayor acceso a la educación ha tenido como consecuencia que una mayor diversidad de alumnos acceda a la escuela; sin embargo, los actuales sistemas educativos se caracterizan por ofrecer respuestas homogéneas a realidades, contextos y necesidades diversas, generando exclusión y discriminación.

Las políticas de equidad están condicionadas por la concepción que se tenga de la diferencia; como algo que se desvía de lo normal o como algo normal que nos enriquece. Muchos programas o acciones tienen un enfoque más centrado en el déficit; es decir, en lo que le falta a ciertos grupos de personas (pobres, indígenas, personas con necesidades educativas especiales, etc.), por ello las políticas proporcionan ayudas y recursos compensatorios que les permitan llegar a un punto idéntico respecto a quienes no tienen ese déficit. Esta perspectiva corre el peligro de confundir diversidades con desigualdades.

La respuesta a la diversidad implica transitar desde un enfoque homogeneizador en el que se ofrece lo mismo a todos, y refleja las aspiraciones de las culturas y clases dominantes, a uno que considere las distintas identidades, necesidades y opciones de las personas y valore las diferencias como algo que enriquece a las personas y sociedades (OREALC/UNESCO, 2007a). En este tránsito hay que tener cuidado en exaltar demasiado lo que nos diferencia porque esto puede conducir a la intolerancia, la exclusión, o a posturas fundamentalistas, justificando por ejemplo la necesidad de un currículo diferente para la población indígena o con discapacidad. Todo ello no haría más que intensificar la actual fragmentación social y cultural que caracteriza a nuestras sociedades.

La pregunta clave entonces es ¿cómo conseguir la igualdad respetando al mismo tiempo las diferencias? La orientación debería ser que existan unos principios, orientaciones y aprendizajes comunes para todos, que aseguren la igualdad de oportunidades, definiendo al mismo tiempo recursos y estrategias diferenciadas, pero no excluyentes o discriminatorias, para atender las necesidades educativas específicas de determinadas personas o grupos. Por ejemplo, la respuesta a la diversidad cultural implica que la educación tenga una dimensión intercultural para todos y el conocimiento en profundidad de la propia cultura y el bilingüismo para aquellos que provienen de pueblos originarios, a través de métodos de enseñanza culturalmente apropiados. Los alumnos que aprenden en su lengua materna durante un periodo de 6 a 8 años, consiguen mejores resultados que los que empiezan su aprendizaje en la lengua oficial (UNESCO, 2007).

En el caso de los estudiantes con necesidades educativas especiales es preciso adaptar el currículo y las actividades educativas y brindarles el apoyo de los especialistas que requieran para optimizar su proceso de desarrollo y aprendizaje. Los niños de familias migrantes también requieren acciones específicas para la inmersión cultural y lingüística del país de acogida, y la igualdad de género exige eliminar los estereotipos en las expectativas de los docentes, en los materiales y en las actividades que se proponen. 


\section{Avanzar desde políticas basadas prioritariamente en los factores objetivos hacia políticas centradas en los sujetos}

Las reformas educativas implementadas han estado centradas en promover cambios en las estructuras y los insumos, elaborando nuevos currículos, aumentando el tiempo de aprendizaje, mejorando la infraestructura escolar, modernizando los ministerios de educación, o proporcionando computadoras y libros de textos. Si bien estos aspectos son importantes para mejorar la calidad de la educación han sido insuficientes para lograr mejores aprendizajes y promover cambios significativos en las aulas, en las interacciones entre docentes y alumnos y de estos entre sí.

Diferentes estudios realizados en escuelas de contextos difíciles que logran buenos resultados han mostrado que los factores que hacen la diferencia tienen que ver con factores subjetivos; compromiso y motivación de los docentes, relaciones horizontales entre directivos y docentes, docentes y padres que tienen altas expectativas sobre los estudiantes, y buen clima, entre otros (OREALC/ UNESCO, 2002). Esto nos lleva a la necesidad de desarrollar políticas que presten mayor atención a la dimensión subjetiva de los diferentes actores involucrados en los procesos educativos, incrementando sus capacidades y motivaciones y mejorando sus condiciones de trabajo.

La formación de los docentes debería prepararles para trabajar en distintos contextos y realidades y proporcionarles herramientas para atender la diversidad en todos los niveles educativos, como la adaptación del currículo, la utilización de distintas estrategias de enseñanza, la identificación de barreras para el aprendizaje y la participación, la incorporación de la cultura a los procesos educativos, o el trabajo colaborativo con otros docentes y las familias, entre otros aspectos. Sin embargo, por muy capaz que sea un docente, algunas necesidades educativas son muy complejas y específicas y requieren la intervención de otros profesionales con conocimientos más especializados. En algunos casos se requieren especialistas que puedan abordar las necesidades educativas especiales, o profesores que puedan atender las necesidades de niños con otras lenguas y culturas, o que puedan atender las necesidades específicas de niños que viven en circunstancias de vida difíciles o son portadores de VIH/SIDA.

\section{Currículum y procesos pedagógicos pertinentes y centrados en las necesidades de todos los estudiantes}

Uno de los elementos fundamentales de la inclusión es promover la máxima participación de los estudiantes en el currículo y las actividades educativas para que tengan éxito en su aprendizaje. Para que esto sea posible el currículo debiera reunir las siguientes características: tener el suficiente grado de apertura y flexibilidad para que se pueda adaptar a las diferentes necesidades de los estudiantes y de los contextos en los que se desarrollan; contemplar de forma equilibrada las competencias necesarias para ejercer la ciudadanía mundial y local y el desarrollo de las múltiples inteligencias; tener una dimensión intercultural para todos y considerar el bilingüismo y el conocimiento de la 
propia cultura para los estudiantes de pueblos originarios; y contemplar aprendizajes orientados a convivir juntos en el respeto a la singularidad de cada uno.

Dar respuesta a la diversidad significa romper con el esquema tradicional en el que todos los niños hacen lo mismo, de la misma forma y con los mismos materiales. La cuestión central es cómo organizar las situaciones de enseñanza de forma que sea posible personalizar las experiencias de aprendizaje comunes; es decir, cómo lograr el mayor grado posible de interacción y participación de todos los alumnos, sin perder de vista las necesidades concretas de cada uno. Para ello es preciso conocer en profundidad a todos los estudiantes, utilizar una amplia variedad de estrategias metodológicas y adaptar las tareas de aprendizaje a sus posibilidades (Blanco, 1999).

\section{Considerar las diferencias en los sistemas nacionales de evaluación de la calidad}

Una de las barreras más importantes para avanzar hacia la inclusión tiene que ver con la evaluación, la promoción y la acreditación. Una cuestión crucial es cómo conciliar una enseñanza respetuosa de las diferencias y de los procesos individuales de aprendizaje con una evaluación que termina siendo igual para todos. Desde la perspectiva de una educación inclusiva el fin de la evaluación no es clasificar o etiquetar a los alumnos, sino identificar el tipo de ayudas y recursos que precisan para facilitar su proceso de aprendizaje y optimizar el pleno desarrollo de sus capacidades. Esto significa evaluar tanto las dificultades y potencialidades del alumno como los factores de la enseñanza y del contexto educativo y familiar que pueden estar favoreciendo o dificultando su aprendizaje y participación.

Los actuales sistemas nacionales de medición de la calidad son también una barrera para la educación inclusiva, especialmente cuando se establecen comparaciones entre escuelas y se otorgan incentivos basados en los resultados de aprendizaje de los estudiantes. Esta situación conlleva que muchas escuelas, especialmente las privadas, excluyan a aquellos alumnos que pueden tener menor nivel de logro y por tanto pueden bajar la puntuación de la escuela.

El desafío es avanzar hacia sistemas de evaluación que se preocupen de identificar no sólo los logros de estudiantes sino también los factores que están limitando su aprendizaje y el desarrollo de la institución educativa, con el fin de proporcionar a cada escuela los recursos y apoyos que requiere para atender las necesidades de todo el alumnado. Los procesos de evaluación deberían considerar las diferencias elaborando pruebas en diferentes lenguas o en Braille, cautelando que los ítemes sean significativos desde el punto de vista cultural y de género, y ofreciendo a los estudiantes con necesidades educativas especiales las ayudas que les permitan expresar su verdadero grado de conocimiento como, por ejemplo, darles más tiempo para hacer las pruebas o tener un intérprete en lenguaje de señas.

Los desafíos señalados precisan una fuerte voluntad política y el apoyo del conjunto de la sociedad para hacer posible una educación para todos, con todos y para cada uno, que es la aspiración del movimiento de la inclusión. 


\section{Bibliografía}

Ainscow, M. (2001). El desarrollo de escuelas inclusivas. Madrid: Narcea.

Artigas, C. (2003). La incorporación del concepto de derechos económicos, sociales y culturales al trabajo de la CEPAL. Serie Políticas Sociales. Santiago de Chile: CEPAL.

Blanco, R. (1999). La atención a la diversidad en el aula y las adaptaciones del currículo. En A. Marchesi, C. Coll y J. Palacios (comp.). Desarrollo psicológico y Educación III. Necesidades educativas especiales y aprendizaje escolar (pp. 411436). Madrid: Alianza.

Blanco, R. (2006). La equidad y la inclusión social: uno de los desafíos de la educación y la escuela hoy. Revista electrónica Iberoamericana sobre Calidad y Cambio en Educación, 3(4), 1-15. Disponible en: http:// www.rinace.net/vol4num3.htm.

Blanco, R. (en prensa). Construyendo las bases de la inclusión y la calidad de la educación en la primera infancia. Revista de Educación. Monográfico 347 sobre Atención socioeducativa a la primera infancia, coordinado por la doctora María Paz Lebrero Baena. Madrid: Universidad Complutense de Madrid.

Bоотн, T. y Ainscow, M. (2000). Índice de inclusión: Desarrollando el aprendizaje y la participación en las escuelas. Bristol: CSIE.

Boотн, T. y Ainscow, M. (2004). Índice de inclusión: Desarrollando el aprendizaje y la participación en las escuelas. (Ana Luisa López, trad.). Santiago de Chile: Oreald UNESCO.

Coll, C. y Martin, E. (2006). La vigencia del debate curricular. El currículo a debate. Revista PRELAC, 3, 6-28.

GARCÍA-Huidobro, J. (2005, diciembre). La igualdad en educación como bien democrático y de desarrollo. Ponencia pre- sentada en la Reunión del Comité Intergubernamental del PRELAC, organizada por Unesco/Orealc, Santiago de Chile.

Marchesi, A. y Martin, M. (1998). Calidad de la enseñanza en tiempos de cambio. Madrid: Alianza.

OECD (2004). Learning for Tomorrow's world. First results from Pisa 2003. Programme for International Students Assessment. Paris: OECD.

ONU (1965). Convención Internacional sobre la eliminación de todas las formas de discriminación racial. Disponible en: http:// www.unhchr.ch/spanish/html/menu3/b/d_ icerd_sp.htm.

ONU (1979). Convención sobre la eliminación de toda forma de discriminación contra la mujer. Disponible en: http://www. unhchr.ch/spanish/html/menu3/b/e1cedaw_sp.htm.

ONU (1992). Declaración sobre los derechos de las personas pertenecientes a minorías nacionales o étnicas, religiosas y lingüísticas. Disponible en: http://www.unhchr.ch/spanish/html/menu3/b/d_minori_sp.htm.

OnU (2006). Convención de los derechos de las personas con discapacidad. Disponible en: http://www.un.org/disabilities/documents/convention/convoptprot-s.pdf. Onu/Ecosoc/UnesCo (2003). Right to Education. Scope and Implementation. General comment 13 on the right to Education (article 13 of the International covenant on economic, social and cultural rights). París: UNESCO.

Orealc/Unesco (2001). Informe técnico. Primer estudio Internacional comparativo sobre lenguaje, matemática y factores asociados para alumnos de tercer y cuarto grado de la educación básica. Santiago de Chile: Orealc/Unesco/Llece. 
Orealc/Unesco (2002). Estudio cualitativo de escuelas con resultados destacables en siete países latinoamericanos. Santiago de Chile: Orealc/Unesco/Llece.

Orealc/Unesco (2007a). Educación de calidad para todos: un asunto de derechos humanos. Documento de discusión sobre políticas educativas en el marco de la II Reunión Intergubernamental del Proyecto Regional de Educación para América Latina y el Caribe (EPT/PRELAC). Santiago de Chile: Orealc/Unesco.

Orealc/Unesco (2007b). Situación educativa de América Latina y el Caribe: garantizando la educación de calidad para todos. Informe regional de revisión y evaluación del progreso de América Latina y el Caribe hacia la EPT. Santiago de Chile: OrEald UNESCO.

SCHMELKes, S. (2005). La educación intercultural en México. En J. García-Huidobro (Ed.). Políticas educativas y equidad. Reflexiones del Seminario Internacional (pp. 185-189). Santiago de Chile: Fundación Ford- Universidad Alberto HurtadoUNICEF-UNESCO.

SEN, A. (1999, marzo). Invertir en la infancia: su papel en el desarrollo. Ponencia presentada en la Asamblea Anual del Banco Interamericano de Desarrollo. Romper el ciclo de la pobreza: Invertir en la infancia, París.

Tedesco, J. (2005). Igualdad de oportunidades y política educativa. En J. García-Huidobro (ed.). Políticas educativas y equidad. Reflexiones del Seminario Internacional (pp. 59-68). Santiago de Chile: Fundación Ford-Universidad Alberto Hurtado-Unicef-Unesco.

Tomasevski, K. (2003). Contenido y vigencia del Derecho a la Educación. Cuadernos
Pedagógicos, 36, 15-38. San José de Costa Rica: IIDH.

ToMASEvsKi, K. (2006). The state of the right to education worldwide. Free or fee: 2006 Global report. Disponible en: http:// www.katarinatomasevski.com/images/ Global_Report.pdf.

UNESCO (1960). Convention against Discrimination in Education. Disponible en: http://unesdoc.unesco.org/ images/0013/001325/132598e.pdf Unesco (1994). Conferencia Mundial de Salamanca sobre Necesidades educativas especiales: acceso y calidad. Salamanca: UNESCO.

UNESCO (1996). La educación encierra un tesoro. Informe de la UnEsCo de la Comisión Internacional sobre la Educación para el siglo XXI, presidida por Jacques Delors. París: UNESCO.

Unesco. (2004). Financiamiento de la educación de la primera infancia: ¿Qué conviene saber? Notas de la UNESCO sobre las políticas de la primera infancia, 23.

UnESCO (2005). Guidelines for inclusion. Ensuring acces to education for all. París: UNESCO.

UNESCO (2007). Educación para todos: Bases sólidas. Atención y educación de la primera infancia. Informe de seguimiento de Educación para Todos en el Mundo. París: UNeSCo.

Van Der GaAG, J. (2000). From early child development to human development. En M. Young (ed.). From early child development to human development: investing in our children's future (pp. 63-78). Washington: The World Bank. 